\title{
The restricted primitive model of ionic fluids with nonadditive diameters
}

\author{
Riccardo Fantoni ${ }^{1}$ and Giorgio Pastore ${ }^{2}$
}

1 Dipartimento di Scienze dei Materiali e Nanosistemi, Università Ca' Foscari Venezia, Calle Larga S. Marta DD2137, I-30123 Venezia, Italy

${ }^{2}$ Dipartimento di Fisica dell' Università and IOM-CNR, Strada Costiera 11, 34151 Trieste, Italy

\author{
PACS 68.43.Hn - \\ PACS $61.20 . \mathrm{Qg}-$ \\ PACS $64.70 . \mathrm{pv}-$ \\ PACS 64.60.ah- \\ PACS 64.70.F-- \\ PACS 64.60.F- - \\ PACS $64.75 . \mathrm{Yz}-$
}

\begin{abstract}
The restricted primitive model with nonadditive hard-sphere diameters is shown to have interesting and peculiar clustering properties. We report accurate calculations of the cluster concentrations. Implementing efficient and ad hoc Monte Carlo algorithms we determine the effect of nonadditivity on both the clustering and the gas-liquid binodal. For negative nonadditivity, tending to the extreme case of completely overlapping unlike ions, the prevailing clusters are made of an even number of particles having zero total charge. For positive nonadditivity, the frustrated tendency to segregation of like particles and the reduced space available to the ions favors percolating clusters at high densities.
\end{abstract}

Ionic soft matter [1,2] is a class of conventional condensed soft matter whose interactions are dominated by - electrostatics crucially affecting its physical properties. Among the most popular representatives of such a class of materials are natural and synthetic saline environments, like aqueous and non-aqueous electrolyte solutions and - molten salts, including room-temperature ionic liquids, as well as a variety of polyelectrolytes and colloidal suspensions. Equally well known are biological systems of proteins.

The simplest fluid modeling an ionic colloidal suspension is the Restricted Primitive Model (RPM) [3] a binary mixture of uniformly charged Hard-Spheres (HS) for which the like-unlike collision diameter between a particle of species 1 , of diameter $\sigma_{11}=\sigma$, and a particle of species 2 of diameter $\sigma_{22}=\sigma$, is equal to the arithmetic mean $\sigma_{12}^{\text {add }}=\left(\sigma_{11}+\sigma_{22}\right) / 2=\sigma$. The two species are of charge $\pm q$ with equal concentrations to ensure charge neutrality, and the particles move in a medium of fixed dielectric constant $\epsilon$. The phase diagram of this model has been widely studied both within computer experiments [4,10] and through analytical theories [11,18].
From these studies emerged how, in the vapor phase of this fluid, and thus in the determination of the phase diagram, an important role is played by association and clustering. In an old paper, [19] one of us studied a more general RPM fluid where it is allowed for size nonadditivity amongst the particles: the like-unlike collision diameter differs from $\sigma_{12}^{\text {add }}$ by a quantity $\Delta=\left(\sigma_{12}-\sigma_{12}^{\text {add }}\right) / \sigma_{12}^{\text {add }}$ called the nonadditivity parameter. It was suggested through the use of integral equation theories, that such a fluid might have a dramatic change of its clustering properties. The nonadditivity of the HS diameters does not destroy the simplifying symmetry of the model but it introduces modifications of the properties of the pure RPM model making it a paradigm for the self-assembly of isotropic particles and a challenge to present day theories of fluids. There seems to be a lack of literature on this subject excepted for Ref. 20].

In this letter, we reconsider such a model fluid by using more direct, highly efficient numerical simulations. In particular we analyze the clustering properties outside of the gas-liquid coexistence region. As we will see the clustering turns out to be greatly affected by the nonadditivity pa- 
rameter. To the best of our knowledge this is the first time that such a model fluid is studied with numerical simulations. The debate on the importance of clustering in the RPM is rejuvenated by studying this new model fluid.

The model system here considered may be realized experimentally through a colloid-star polymer mixture where both species are charged 21, 22 and may be relevant for modeling room temperature ionic liquids [23, 26]. It is the restricted primitive model (RPM) of nonadditive charged hard-spheres (NACHS). The RPM consists of $N / 2$ uniformly charged hard-spheres of diameter $\sigma$ carrying a total charge $+q$ and $N / 2$ uniformly charged hard-spheres of the same diameter carrying a total charge $-q$. The spheres are moving in a dielectric continuum of dielectric constant $\epsilon$. The interaction between ions of apecies $i$ and $j$ a distance $r$ apart is given by

$$
\beta \phi_{i j}(r)=\left\{\begin{array}{ll}
+\infty & r \leq \sigma_{i j} \\
\frac{q_{i} q_{j}}{k_{B} T \epsilon r} & r>\sigma_{i j}
\end{array} \quad, \quad i, j=1,2,\right.
$$

where $\beta=1 / k_{B} T$ with $T$ the absolute temperature and $k_{B}$ the Boltzmann's constant, $q_{i}$ the charge of an ion of species $i$. The ions form a mixture of NACHS, i.e. $\sigma_{11}=\sigma_{22}=\sigma$ and $\sigma_{12}=\sigma(1+\Delta)$, with $\Delta>-1$ the nonadditivity parameter. A thermodynamic state is completely specified by the reduced density $\rho^{*}=\rho \sigma^{3}=N \sigma^{3} / V$, where $V$ is the volume containing the fluid, the reduced temperature $T^{*}=k_{B} T \epsilon \sigma / q^{2}$, and the nonadditivity parameter $\Delta$.

We used canonical NVT Monte Carlo (MC) simulations to study the fluid in a cubic simulation box of volume $V=L^{3}$ with periodic boundary conditions. The long range of the $1 / r$ interaction was accounted for using the Ewald method [27.

We start from a simple cubic configuration of two crystals one made of species 1 and one made of species 2 juxtaposed. The maximum particle displacement, the same along each direction, is determined during the first stage of the equilibration run in such a way to ensure an average acceptance ratio of $50 \%$. We need around $10^{5} \mathrm{MC}$ steps (MCS) in order to equilibrate the samples and $10^{6}$ $\mathrm{MCS} /$ particle for the statistics.

During the simulation we perform a cluster analysis in the vapor phase. After each $100 \mathrm{MCS}$ we determine the number $N_{n}$ of clusters made of $n$ particles, so that $\sum_{n} n N_{n}=N$. We assume 28, 29] that a group of ions forms a cluster if the distance $r$, calculated using periodic boundary conditions, between a particle of species $i$ of the group and at least one other particle of species $j$ is less then some fixed value, i.e. $r<\sigma_{i j}+\delta \sigma$ where $\delta$ is a parameter 11. In all our simulations we chose $\delta=0.1$ (in Ref. [5] a detailed study of the sensitivity of the clustering properties on this parameter is carried out for the pure RPM fluid). Then we take the average of these numbers

${ }^{1}$ Many different ways of defining a cluster have been proposed 12 15 30,32 since the Bjerrum theory 33. 30 of ionic associations first appeared. Our choice corresponds to the geometric one of Gillan 12 . $\left\langle N_{n}\right\rangle$. Here $Q_{n}=n\left\langle N_{n}\right\rangle / N$ gives the probability that a particle belongs to a cluster of size $n$. To establish a criterion for percolation, we also find clusters without using periodic boundary conditions. One of these clusters percolates if, amongst its particles, there are two that do not satisfy the cluster condition as a pair, but do satisfy the condition if periodic boundary conditions are used.

In Fig. 11 we simulated the fluid at a temperature $T^{*}=0.1$ above the critical temperature, $T_{c}^{*} \approx 0.05$, of the pure RPM 6, 10. We see how, at high density, a positive nonadditivity is responsible for a gain of clustering in the fluid, which tends to admit percolating clusters also due to the fact that a positive nonadditivity pushes the fluid at densities closer to the maximum density attainable. It is well known that in the neutral nonadditive hard-sphere fluid a positive nonadditivity tends to demix the mixture at lower densities as $\Delta$ increases [34 39, so in our fluid we will have a competition between the tendency to demix in the neutral nonadditive hard-sphere fluid and the tendency to cluster in the RPM fluid. At $\rho^{*}=0.45$ both the pure RPM and the $\Delta=+0.3$ have percolating clusters. Lowering the density we first reach a state, at $\rho^{*}=0.3$, where the negative nonadditivity gives the same clustering as the RPM and the positive nonadditivity gives higher percolating clustering, then a state, at $\rho^{*}=0.1$, where the positive nonadditivity gives the same clustering of RPM and the negative nonadditivity a higher one, and finally a state, at $\rho^{*}=0.01$, at low densities where a negative nonadditivity increases the clustering over the RPM fluid and a positive nonadditivity diminishes it. Summarizing, for the fixed values of $|\Delta|$ used, we find, in agreement with Ref. [19, that: (a) at high density and positive $\Delta$ we have more clustering than in the additive model, (b) at high density and negative $\Delta$ we have less clustering than in the additive model, (c) at low density and positive $\Delta$ we have less clustering than in the additive model, (d) at low densities and negative $\Delta$ we have more clustering than in the additive model. These points can be explained observing that a pair of unlike ions have a higher affinity with negative $\Delta$. Thus, in a bulk phase negative $\Delta$ favors etherocoordination. Clusters of a given number of ions tend to be smaller when $\Delta$ is negative. As a result, at low density (where excluded volume plays a small role), the extra affinity due to negative $\Delta$ enhances cluster formation. By contrast, at high densities, the increase in available volume from the resulting etherocoordination with negative $\Delta$ has an important role, reducing the density-driven imperative to form clusters in the negative $\Delta$ case. The same arguments in reverse explain the behavior of a system with positive nonadditivity where now homocoordination at high density is favored [19].

To qualitatively reproduce the curves with nonpercolating clusters we can use the Tani and Henderson clustering analysis [28, 29, 40, with an inter-cluster configurational partition function the one of an ideal gas of clusters, in reduced units, $Z_{\text {inter }} \approx\left(V / \sigma^{3}\right)^{N_{t}}$, where $N_{t}=\sum_{n=1}^{n_{c}} N_{n}$ is the total number of clusters and we 
assume to have only clusters made of up to $n_{c}$ particles. Then the equations for the equilibrium cluster concentrations are

$$
\begin{aligned}
\left\langle N_{n}\right\rangle / N & =\lambda^{n} z_{n}^{\text {intra }} / \rho^{*}, \quad n=1,2, \ldots, n_{c} \\
1 & =\sum_{n=1}^{n_{c}} n\left\langle N_{n}\right\rangle / N
\end{aligned}
$$

where $z_{n}^{\text {intra }}$ are the configurational intra-cluster partition functions in reduced units with $z_{1}^{\text {intra }}=2$ and $\lambda\left(=\alpha \rho^{*} / 2\right)$ is a Lagrange multiplier to be determined by Eq. (3). Moreover neglecting the excess internal energy of the clusters we can approximate $z_{n}^{\text {intra }} \approx\left(v_{n} / \sigma^{3}\right)^{(n-1)} 2^{n} / n$ ! where $v_{n}$ is the volume of an $n$-cluster. Assuming further the cluster to be in a closed packed configuration we can approximate $2 v_{n} \approx n \sigma^{3} / \sqrt{2}$. This simple approximation is temperature independent and its usefulness is thereby quite limited.

We checked the size dependence of the curves shown in Fig. 1 and saw that when we have no percolating clusters the curve was unaffected by a choice of an higher number of particles (up to 5000), while the curve changed in presence of percolating clusters. In this case we found that a common curve is given by $\left\langle N_{x}\right\rangle / N$ with $x=n / N \in[0,1]$. Then, in order to satisfy the normalization condition, $1=\sum_{n} n\left(\left\langle N_{n}\right\rangle / N\right) \approx \int d x x N^{2}\left(\left\langle N_{x}\right\rangle / N\right)$, we must have $\left(\left\langle N_{x}\right\rangle / N^{\prime}\right) /\left(\left\langle N_{x}\right\rangle / N^{\prime \prime}\right) \approx\left(N^{\prime \prime} / N^{\prime}\right)^{2}$ for two different sizes $N^{\prime}$ and $N^{\prime \prime}$.

In Fig. 2 we show the clustering analysis for the fluid with $\Delta$ approaching -1 at $T^{*}=0.1$ and $\rho^{*}=0.45$. We see how letting $\Delta$ approach -1 this stabilizes small neutrally charged clusters and lowers the degree of dissociation $\alpha=\left\langle N_{1}\right\rangle / N$. The first stable cluster is the dipole: the "overlap" of a positive and a negative sphere. This are dipoles of moment $q r_{12}$ with $r_{12}<\sigma(1+\Delta+\delta)$ which may lack a gas-liquid criticality [41]. We clearly have a transition from a conducting to an insulating phase as $\Delta$ goes from 0 to -1 . We expect that in the limiting case of $\Delta=-1$ the system we obtain is the neutral HS fluid of half the density. This is confirmed by a comparison of the like radial distribution functions with the one of the neutral HS even if the $\Delta=-1$ fluid simulation rapidly slows down into the frozen configuration of the overlapping anions and cations. In order to overcome this problem one should alternate single particle moves with neutrally charged 2 -cluster moves.

In order to qualitatively reproduce the curve of Fig. 2 we need to use Eqs. (2)-(3) with $z_{n}^{\text {intra }}=\sum_{s=0}^{n} z_{s, n-s}^{\text {intra }}$ where $z_{s, t}^{\text {intra }}$ are the configurational intra-cluster partition

${ }^{2}$ Clearly a proper analysis of the cluster volume would itself require a MC simulation [12. functions of a cluster made of $s$ anions and $t$ cations,

$$
\begin{aligned}
z_{s, t}^{\text {intra }}= & \frac{1}{s ! t !} \int_{\Omega_{s, t}} \frac{d \mathbf{r}_{2} \ldots d \mathbf{r}_{s+t}}{\sigma^{3(s+t-1)}} \times \\
& e^{-\beta \sum_{\mu>\nu=1}^{s+t} \phi_{i_{\mu} j_{\nu}}\left(r_{\mu \nu}\right)} \\
\approx & \frac{(s+t)^{(s+t-1)}}{s ! t !}\left(K / K_{0}\right)^{\min \{s, t\}}, \\
K / K_{0}= & \int_{\sigma(1+\Delta)}^{\lambda_{B} / 2} r^{2} e^{+\lambda_{B} / r} d r / \int_{\sigma(1+\Delta)}^{\lambda_{B} / 2} r^{2} d r,
\end{aligned}
$$

where the configurational integral goes only over the relative positions and it covers the region $\Omega_{s, t}$ of $s$ anions clusters configuration space, $\lambda_{B}=\sigma / T^{*}$ is the Bjerrum length, Roman indeces denote the particle species, Greek indeces denote the particle labels, a Roman index with a Greek subindex denotes the species of the particle corresponding to the Greek subindex, and $\mathbf{r}_{\mu \nu}$ denotes the separation vector between particle $\mu$ and particle $\nu$. Eq. (5) is justified as follows. Let us call the anions $i_{-}=1_{-}, \ldots, s_{-}$ and the cations $j_{+}=1_{+}, \ldots, t_{+}$. From Eq. (4) follows

$$
\begin{aligned}
z_{t, t}^{\text {intra }}= & \frac{1}{t !^{2}} \frac{1}{\sigma^{3(2 t-1)}} \int_{\Omega_{t, t}} \prod_{l=2}^{t} d \mathbf{r}_{1_{+} l_{-}} \prod_{k=1}^{t} d \mathbf{r}_{k_{+} k_{-}} \\
& \times \prod_{i>j=1}^{t} e^{-2 \lambda_{B} / r_{i_{+} j_{+}}} \prod_{i, j=1}^{t} e^{+\lambda_{B} / r_{i_{+} j_{-}}} \\
\approx & \frac{1}{t !^{2}} \frac{1}{\sigma^{3(2 t-1)}} \int_{\Omega_{t, t}} \prod_{l=2}^{t} d \mathbf{r}_{1_{+}} l_{-} \prod_{k=1}^{t} d \mathbf{r}_{k_{+} k_{-}} \\
& \times \prod_{i, j=1}^{t} e^{+\lambda_{B} / r_{i_{+} j_{-}}},
\end{aligned}
$$

where we approximated $e^{-\lambda_{B} / r} \approx 1$ which is justified at high $T^{*}<1 / 2(1+\Delta)$ or low $\lambda_{B}$. Now we observe that for example $r_{1_{+} 2_{-}}=\left|\mathbf{r}_{1_{+} 1_{-}}+\mathbf{r}_{1_{-} 2_{-}}\right|$with $r_{1_{-} 2_{-}}>\sigma$ and $e^{+\lambda_{B} / r_{1}{ }^{2}-} \approx 1$. So that for negative nonadditivity we can further approximate

$$
\begin{aligned}
& z_{t, t}^{\text {intra }} \approx \frac{1}{t !^{2}} \frac{1}{\sigma^{3(2 t-1)}} \int_{\Omega_{t, t}} \prod_{l=2}^{t} d \mathbf{r}_{1_{+}} l_{-} \prod_{k=1}^{t} d \mathbf{r}_{k_{+} k_{-}} \\
& \times \prod_{i, j=1}^{t} e^{+\lambda_{B} / r_{i_{+} j_{-}}} \\
& \approx \frac{1}{t !^{2}} \frac{1}{\sigma^{3(2 t-1)}} \int_{\Omega_{t, t}} \prod_{l=2}^{t} d \mathbf{r}_{1_{+} l_{-}} \prod_{k=1}^{t} d \mathbf{r}_{k_{+} k_{-}} \\
& \times \prod_{i=1}^{t} e^{+\lambda_{B} / r_{i_{+} i_{-}}} \\
& \stackrel{(2 t)^{(2 t-1)}}{t !^{2}}\left(K / K_{0}\right)^{t}
\end{aligned}
$$

where the factor $(2 t)^{(2 t-1)}$ takes into account the volume of $\Omega_{t, t}$. Using the same chain of approximations we reach Eq. (5). We immediately see how $z_{1,1}^{\text {intra }} \propto K / \sigma^{3}$ becomes 

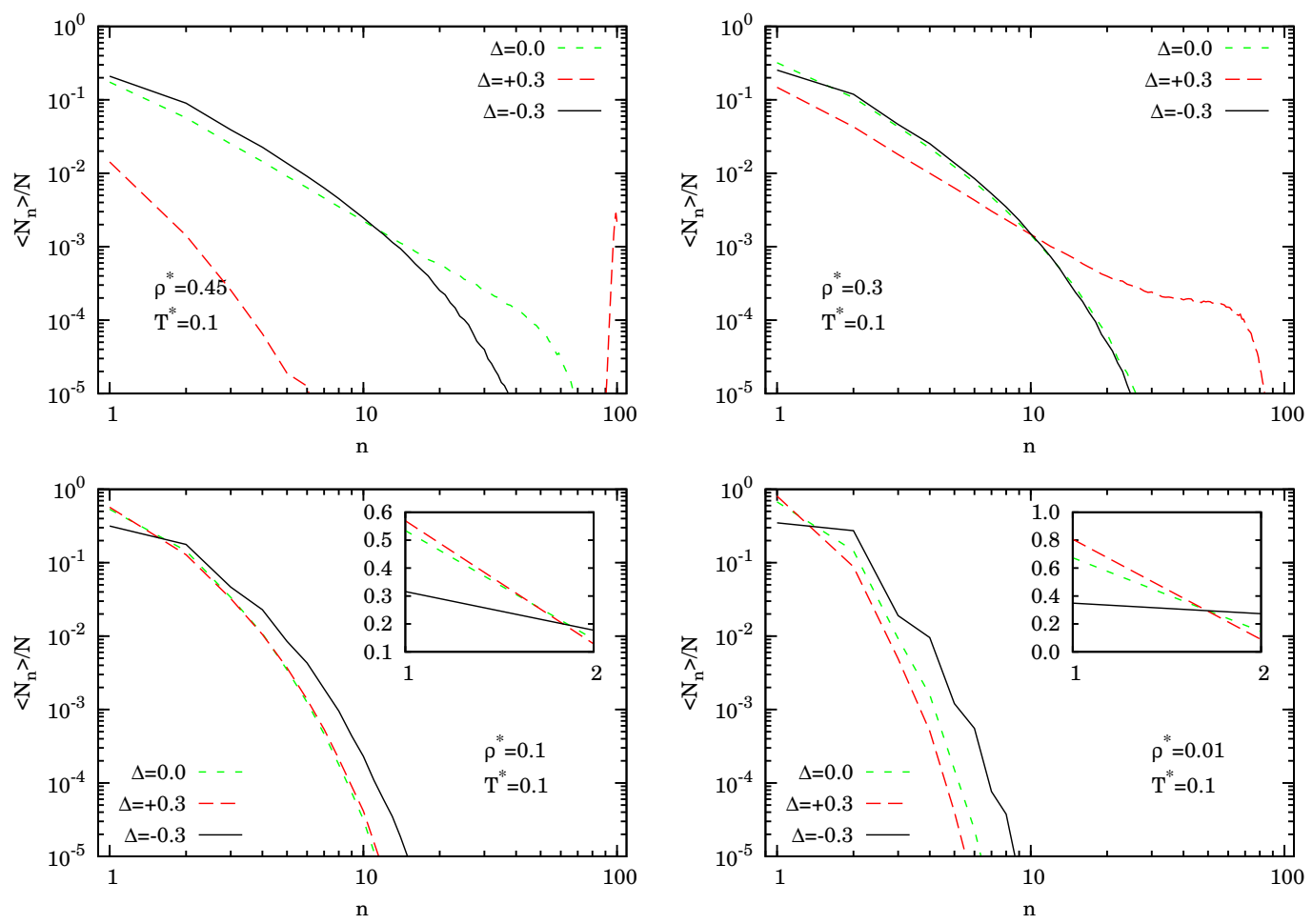

Fig. 1: (color online). Clustering properties of the fluid at various values of nonadditivity and density. $N_{n}$ are the number of clusters made of $n$ particles. We chose $\delta=0.1$. In the MC simulations we used $N=100$ particles and a number of MCS $=10^{7}$. The insets allow $\alpha=\left\langle N_{1}\right\rangle / N$, the degree of dissociation, to be directly read-off from the graph.

bigger and bigger as $\Delta \rightarrow-1$ and the same holds for all the $z_{k, k}^{\text {intra }}$ which clearly dominate over all the others $z_{s, t}^{\text {intra }}$ with $s \neq t$. And this qualitatively explains Fig. 2

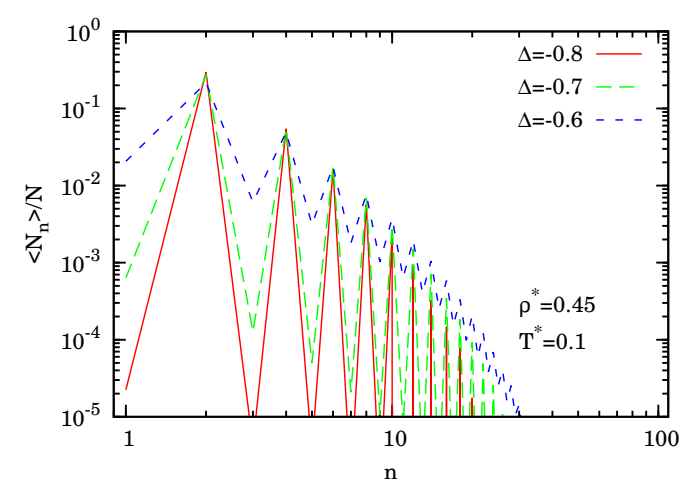

Fig. 2: (color online). We show the clustering properties of the fluid at $T^{*}=0.1$ and $\rho^{*}=0.45$ at various values of negative nonadditivity approaching $-1 . N_{n}$ are the number of clusters made of $n$ particles. We chose $\delta=0.1$. In the MC simulations we used $N=100$ particles and a number of $\mathrm{MCS}=5 \times 10^{7}$.

Sufficiently close to the critical point we determined the qualitative change in the behavior of the gas-liquid coexistence region by switching on a negative or a positive nonadditivity. To this aim we divided the simu- lation box into $m^{3}$ cubes of side $\ell=L / m$ and registered the density inside each cell $\rho_{i}=\mathcal{N}_{i} / \ell^{3}$, where $\mathcal{N}_{i}$ is the number of particles inside the $i$ th cell so that $\sum_{i=1}^{m^{3}} \mathcal{N}_{i}=N$. Then we calculated the density distribution function $P_{m}(\rho)=\sum_{i=1}^{m^{3}} P_{m}\left(\rho_{i}\right) / m^{3}$,42, 43, where $P_{m}\left(\rho_{i}\right)$ is the distribution function for the $i$ th cell. With $\int P_{m}(\rho) d \rho=1$. Above the critical temperature the density probability distribution function can be described by a Gaussian centered at the simulation density whereas below it becomes bimodal with two peaks one centered at the gas density and one at the liquid density.

We start from an initial configuration of particles of random species placed on a simple cubic lattice. We equilibrate (melt) the fluid for $10^{6} \mathrm{MCS} /$ particle. We then sampled the distribution function every 10 MCS. To enhance the efficiency of the determination of the cell density distribution, every $10 \mathrm{MCS}$, we choose the subdivision of the simulation box in cells with a random displacement $\mathbf{r}=\left(r_{x}, r_{y}, r_{z}\right)$ with $r_{x}, r_{y}, r_{z} \in[0, L]$. And we measured the distribution function on runs of $10^{6} \mathrm{MCS} /$ particle.

Choosing $m=2$ and $N=100$ the results for the fluid at a temperature $T^{*}=0.02, \rho^{*}=0.2$, well within the coexistence region of the pure RPM fluid, and $\Delta=0, \pm \mathcal{D}$ with $\mathcal{D}=10^{-1}, 10^{-2}, 10^{-3}$ are shown in Fig. 3 In this case the minimum density that can be registered is $1 / \ell^{3}=0.2 \times 8 / 100=0.016$. We see that the pure RPM 
fluid shows a density distribution function which has three peaks with the first peak, which lies below the minimum density, at approximately the low density of the gas phase, the second peak at the simulations density $\rho^{*}=0.2$ which is due to the fact that the fluid develops surfaces between the gas and the liquid phase 44, and the third peak at approximately the high density of the liquid phase. We see from the figure that increasing $\mathcal{D}$ the middle peak is lost first in the positive additive model and then in the negative nonadditive models. Moreover for the biggest $\mathcal{D}$ the peak of the liquid phase is barely visible. This may be due to the fact that one had to choose a proper simulation density closer to the density of the liquid 42,43]. We clearly see how this analysis works like a "microscope" on the degree of nonadditivity predicting an increase(decrease) of the coexistence region for small negative(positive) nonadditivity. This behavior can be explained as follows. Positive nonadditivity increases the effective excluded volume of ions, thereby reducing the density of the liquid phase, and negative nonadditivity does the opposite.

We believe that our results could be relevant for the interpretation of experimental work on the phase diagrams of room temperature ionic liquids 25. In these experimental systems the liquid-liquid binodals shifted above and below the one of the pure RPM are observed depending on the kind of solvent. If on the one hand this can be ascribed to the different dielectric constant of the solvent 24] on the other hand it is clear that depending on the kind of solvent used the anion-cation contact-pairing affinity may vary [45] and thus the different experimental ionic liquids considered should be more correctly described by comparison not just with the pure RPM but with the more realistic primitive model with the addition of either a positive or negative size nonadditivity.

In conclusion, we have performed for the first time a MC simulations study of the vapor phase of the RPM with nonadditive diameters, with particular emphasis on its clustering properties. A density distribution function analysis shows how the gas-liquid coexistence region evolves by switching on the nonadditivity. A negative nonadditivity tends to enlarge the coexistence region while a positive one to shrink it.

From the cluster analysis we where able to distinguish between two kind of behaviors for the cluster concentrations. When we are below the percolation threshold the curves for the cluster concentrations as a function of the cluster size are independent of the number of particles used in the simulation and can be qualitatively explained by a simple clustering theory where one approximates the clusters to form an ideal gas and the $n$-cluster as formed by $n$ non-interacting particles, for not too small density or nonadditivity. When we are above the percolation threshold the curves depend on the number of particles used in the simulation and obey a simple scaling relationship.

At low density, the negative nonadditive fluid has higher clustering than the pure RPM whereas at high densities the positive nonadditive fluid has a greater degree of clus-
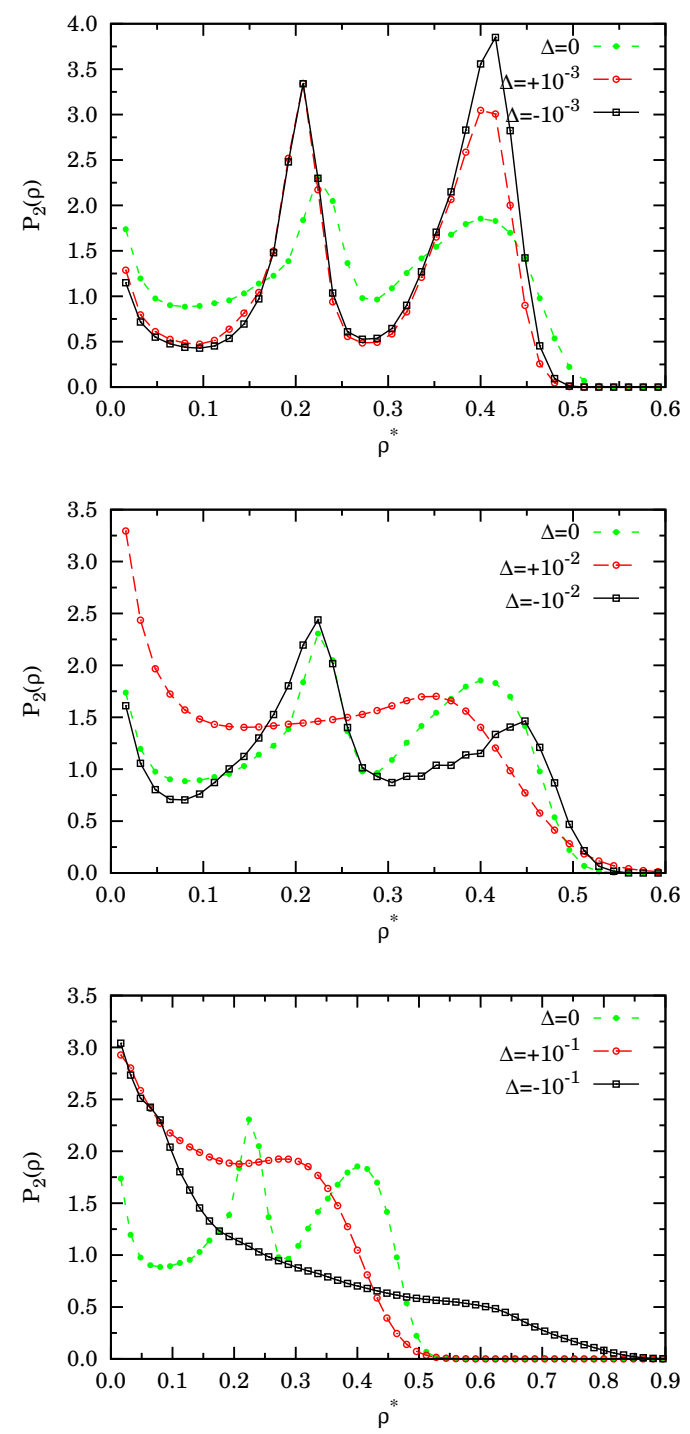

Fig. 3: (color online). Cell density distribution function for the fluid at $T^{*}=0.02, \rho^{*}=0.2$ and $\Delta=0, \pm \mathcal{D}$ with $\mathcal{D}=$ $10^{-3}, 10^{-2}, 10^{-1}$. We used $N=100$ and $m=2$ with $10^{6}$ $\mathrm{MCS} /$ particle.

tering. The positive nonadditive fluid is the first one to reach the percolating clusters upon an increase of the density. This is due to the less space available to the ions, for a given density, for positive nonadditivity and to the frustrated tendency to segregation of like particles at high density. A negative nonadditivity tends to greatly enhance the formation of the neutrally charged clusters, starting with the dipole, as can be predicted from the simple clustering theory refined at the intra-cluster level. Traces of these features can also be read from an analysis of the partial radial distribution function and structure factors, which will be presented elsewhere.

In parallel with the density distribution function analysis we are currently planning to perform a Gibbs ensemble 
MC study of the gas-liquid binodal to establish more accurately the dependence on the nonadditivity parameter.

We hope that the present study could foster additional theoretical and computational studies as well as experimental realizations of these simple but rich fluids.

$$
* * *
$$

R.F. would like to acknowledge the use of the computational facilities of CINECA through the ISCRA call. Both authors would like to thank the referee for useful comments.

\section{REFERENCES}

[1] P. G. De Gennes, Rev. Mod. Phys. , 64 (1992) 645.

[2] D. Henderson, M. Holovko, and A. Trokhymchuk, (Editor) Ionic Soft Matter: Modern Trends in Theory and Applications NATO Science series (Springer, Netherlands) 2004.

[3] J. P. Hansen and I. R. McDonald, Theory of Simple Liquids 2nd Edition (Academic Press) 1986.

[4] G. Orkoulas and A. Z. Panagiotopoulos, J. Chem. Phys., 101 (1994) 1452.

[5] J.-M. Caillol and J.-J. Weis, J. Chem. Phys. , 102 (1995) 7610.

[6] G. Orkoulas and A. Z. Panagiotopoulos, J. Chem. Phys., 110 (1999) 1581.

[7] P. J. Camp and G. N. Patey, J. Chem. Phys., 111 (1999) 9000.

[8] J. M. Romero-Enrique, G. Orkoulas, A. Z. Panagiotopoulos, and M. E. Fisher, Phys. Rev. Lett. , 85 (2000) 4558.

[9] E. Luijten, M. E. Fisher, and A. Z. PanagiotopouLos, Phys. Rev. Lett. , 88 (2002) 185701.

[10] J.-M. Caillol, D. Levesque, and J.-J. Weis, J. Chem. Phys., 116 (2002) 10794.

[11] G. Stell, K. C. Wu, And B. Larsen, Pys. Rev. Lett. , 37 (1976) 1369.

[12] M. J. Gillan, Mol. Phys. , 49 (1983) 421.

[13] J. A. Given, Phys. Rev. A , 45 (1992) 3849.

[14] J. A. Given And G. Stell, J. Chem. Phys. , 96 (1992) 9233.

[15] M. E. Fisher And Y. Levin, Phys. Rev. Lett. , 71 (1993) 3826.

[16] Y. Zhou, S. Yeh, And G. Stell, J. Chem. Phys., 102 (1995) 5785.

[17] J. A. Given And G. Stell, J. Chem. Phys. , 106 (1997) 1195.

[18] J. Jiang, L. Blum, O. Bernard, J. M. Prausnitz, And S. I. Sandler, J. Chem. Phys., 116 (2002) 7977.

[19] G. Pastore, P. V. Giaquinta, J. S. Thakur, and M. P. Tosi, J. Chem. Phys., 84 (1986) 1827 The relationship between our reduced units and their is as follows: $\rho^{*}=$ $3 \sigma^{3} / 4 \pi$ and $T^{*}=\sigma / \Gamma$.

[20] D. M. Zuckerman, Analysis, improvement, and extension of electrolyte theories Ph.D. thesis University of Maryland (1998).

[21] W. C. K. Poon, S. U. EgelhaAf, J. Stellbrink, J. Allgaier, A. B. Schofield, And P. N. Pusey, Phil. Trans. R. Soc. Lond. A , 359 (2001) 897.
[22] W. C. K. Poon, J. Phys.: Condens. Matter, 14 (2002) R859.

[23] H. Weingärtner, M. Kleemeier, S. Wiegand, And W. Schöer, J. Stat. Phys., 78 (1995) 169.

[24] M. Kleemeier, S. Wiegand, W. Schröer, and H. Weingärtner, J. Chem. Phys. , 110 (1999) 3085.

[25] D. Saracsan, C. Rybarsch, and W. Schröer, $Z$. Phys. Chem., 220 (2006) 1417.

[26] W. Schröer and V. R. Vale, J. Phy.: Condens. Matter , 21 (2009) 424119.

[27] M. P. Allen and D. J. Tildesley, Computer Simulation of Liquids (Oxford University Press) 1987.

[28] R. Fantoni, A. Giacometti, F. Sciortino, nd G. PaSTORE, Soft Matter , (2011) 2419.

[29] R. Fantoni, Eur. Phys. J. B , 85 (2012) 108.

[30] J. K. Lee, J. A. Barker, and F. F. Abraham, J. Chem. Phys., 58 (1973) 3166.

[31] W. Ebeling and M. Grigo, Am. Phys. , 37 (1980) 21.

[32] H. L. Friedman and G. Larsen, J. Chem. Phys., 70 (1979) 92.

[33] N. BJerrum, Kgl. Dan. Vidensk. Selsk. Mat.-fys. Medd. , 7 (1926) 1.

[34] M. Rovere and G. Pastore, J. Phys.: Condens. Matter , 6 (1994) A163.

[35] E. Lomba, M. Alvarez, L. L. Lee, and N. G. AlMArza, J. Chem. Phys., 104 (1996) 4180.

[36] K. Jagannathan and A. Yethiraj, J. Chem. Phys., 118 (2003) 7907.

[37] W. T. Góźdź, J. Chem. Phys. , 119 (2003) 3309.

[38] A. Вuнot, J. Chem. Phys., 122 (2005) 024105.

[39] A. Santos, M. López de Haro, and S. B. Yuste, J. Chem. Phys., 132 (2010) 204506.

[40] A. Tani and D. Henderson, J. Chem. Phys., 79 (1983) 2390.

[41] L. Rovigatti, J. Russo, and F. Sciortino, Phys. Rev. Lett. , 107 (2011) 237801.

[42] M. Rovere, D. W. Heermann, and K. Binder, Europhys. Lett. , 6 (1988) 585.

[43] M. Rovere, D. W. Heermann, and K. Binder, J. Phys.: Condens. Matter, 2 (1990) 7009.

[44] B. Smit, Ph. de Smedt, And D. Frenkel, Mol. Phys. , 68 (1989) 931.

[45] I. Kalcher, J. C. F. Schulz, and J. Dzubiella, Phys. Rev. Lett. , 104 (2010) 097802. 